\title{
Experience of Documentation for the Accessibility of Widespread Cultural Heritage
}

\author{
Sandro Parrinello ${ }^{1, *,+}$ and Anna Dell'Amico ${ }^{2, *,+}$ (D) \\ 1 DICAr-Dipartimento di Ingegneria Civile e Architettura, Università di Pavia, 27100 Pavia, Italy \\ 2 DIDA-Dipartimento di Architettura, Università di Firenze, 50121 Florence, Italy \\ * Correspondence: sandro.parrinello@unipv.it (S.P.); anna.dellamico@unifi.it (A.D.) \\ + These authors contributed equally to this work.
}

Received: 28 January 2019; Accepted: 19 March 2019; Published: 25 March 2019

\begin{abstract}
The will to preserve and enhance cultural heritage imposes reflections on databases as containers of information for the accessibility of dispersed heritage. The aim of the research is to validate methodological approaches in order to create 3D models and information systems. These tools improve accessibility to the artifact, according to different interconnection channels, in order to overcome the physical limit imposed by location. Tangible and intangible heritage take the form of a metadata network throughout a critical analysis and elaboration of data. These files create a complex database containing 3D models rich in information that describe cultural systems. The digital survey is the starting point for the development of languages, signs and symbols. It transfers complex objects into virtual systems, developing a multidimensional dialogue capable of integrating actions and meanings. The interactivity of complex databases facilitates the accessibility and disclosure of data to a wide audience. It requires the establishment of representation systems based on the development of virtual environments.
\end{abstract}

Keywords: digital survey; 3D complex database; virtual museum

\section{Introduction}

Cultural heritage is a fundamental resource for the economy and development of society. Its intellectual growth is strictly connected with the assets and activities present within the territory. Dispersed heritage is a natural phenomenon that concerns historical and cultural connections, creating a relationship between tangible and intangible elements through more than physical and geographical connections. This heritage enriches the territory, providing meaning to space. Opposing the natural dispersion of assets, museums and private collections gather, thematize and exalt some aspects of this heritage for communication and didactic purposes. This grouping procedure, on the one hand, increases the asset value of cultural heritage; on the other hand, it empties and impoverishes the territory. There are many examples in history of monuments moved in order to be placed in important museums. This concerns not only works of art but also real buildings placed inside open-air museums (e.g., in the Soviet Union). Dispersed heritage concerns a system of relations and interconnections of communication models disseminated at the territorial level. It crosses physical and administrative boundaries that clarify the formal cultural value of the homogeneous system. However, heritage is affected by an inverse problem today. It is no longer only the territory that divides and fragments the asset, but the cause is mainly the individual cultural organizations (museums, individuals, associations) that manage private collections. Moreover, the various networks of the management of the museum paths regarding the territory often constitute a barrier to the contextualized reading of the asset. Countries such as Italy count many institutions that need to not only preserve their heritage but also manage their accessibility. 
The difficulty of documenting and coherently returning the image of a complex system lies mainly in the management of a large amount of data necessary to describe it. These data, acquired and processed following different methodological approaches, constitute useful information, which risks not establishing a network, which is a dialogue between data and between data and user. New technologies make complex databases interactive systems of remote use, through the implementation and translation of data collected in three-dimensional narrative systems [1]. These allow the user to navigate within a virtual system that reproduces the object and interact directly with the place and with the individual elements that compose it. The virtual environment breaks down barriers and distances, bringing objects and places closer to the reality that is distant and difficult to access, integrating data acquired through surveys, with multimedia and interactive information. Through the development of supports based on virtual reality and augmented reality, information acquired and cataloged for educational purposes become accessible and usable [2]. This makes it possible to broaden the idea of the museum, which is no longer rooted in the material concept of the building as a container, limited by the obligatory paths of the exhibition rooms (Figure 1). It is transformed into a double of reality, an information machine, where exhibition possibilities and the interconnections between the documents become multiple, integrating and expanding the real experience of the visitor [3].

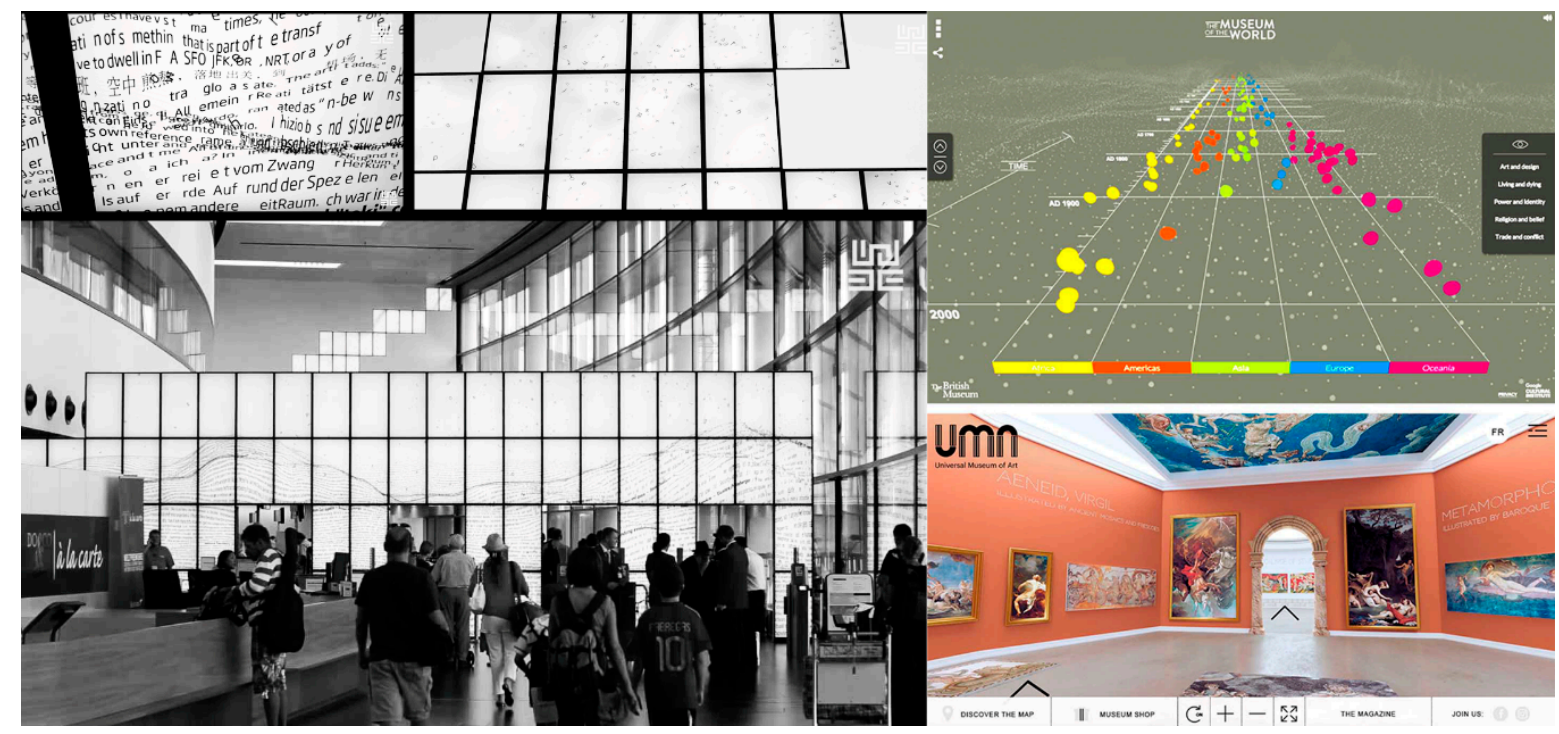

Figure 1. On the left, Textscapes, an installation for the new Vienna Airport by Ars Electronica Future $L a b$, provides a cultural landscape entirely built from falling letters and ideograms belonging to all the languages of the world. As the letters fall, they make sounds that change in volume according to the number of people passing by-a virtual landscape that extends to infinity, symbolizing the air traffic. (Images taken from the short video "ZeitRaum-making of" published on Youtube by Ars Electronic Future Lab). On the right, two examples of digital museum archives, the Museum of the world (sponsored by the British museum) on the top right and the UMA virtual architectural space, on the bottom right, explicate the connections between the findings and make possible access to information about the historical findings.

It is an example of the project born in 2003 from the collaboration between Consiglio Nazionale delle Ricerche (CNR), the Ministry of Foreign Affairs and the National Museum of Baghdad. During the Iraq conflict, the museum suffered a lot of damage; in addition to the structures, many artifacts were stolen. The project, in addition to restoring the premises for the rehabilitation of the museum, has implemented the digital transposition of some works in the museum collection, enclosing them within a digital space, the Virtual Museum of Iraq (Figure 2).

The virtual museum has become a complementary space of the real museum, where it is possible to observe in digital format the three-dimensional models of Iraq artistic heritage enriched by audio 
and video contents. The website has assumed an even more significant value and role when the Museum of Baghdad, due to the political criticality for a first period, was not reopened to all visitors; only the Iraqi schoolchildren could access the museum halls. However, the digital version has been decisive in making the national historical-archaeological patrimony accessible all over the world, as well as being a useful support tool for having a definite catalog of the museum's works which is useful for the investigation of intelligence services engaged in the count and in the discovery of the stolen objects. In this way, the virtual has become a really useful tool and a fundamental element of the identity of Iraq [4].
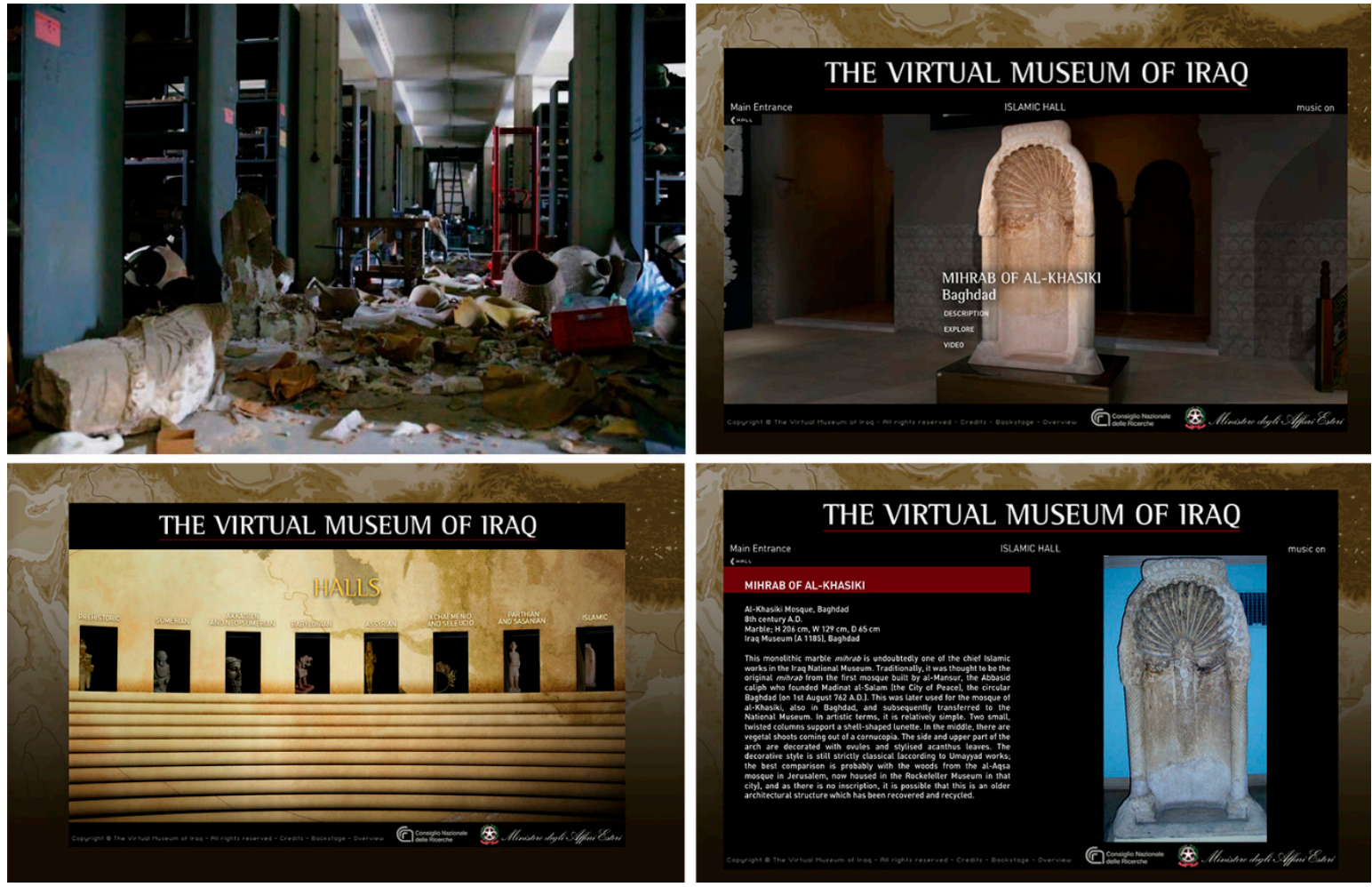

Figure 2. Starting from the top left, a photograph published on the website https:// archeologiavocidalpassato.wordpress.com/tag/museo-di-baghdad/ of the situation of the museum after the events of 2003. On the bottom left the main home page of the website of the virtual mueum of Iraq and on top right and bottom right some screens about the interface during the virtual visit of the virtual museum where the user can discover the digitized objects.

This way accessing the cultural asset also allows protecting it; making the virtual archetype interactive means it can be approached and manipulated without placing the original artifact at risk. Sometimes it is, therefore, paradoxically more useful in the virtual environment rather than in reality. The possibility of interacting with the object also makes learning and knowledge more concrete.

The advancement of computer literacy has led to a profound transformation of society; the transposition of real life into a virtual hypertext formed by a network of services that form a no man's land accessible to all has led to a radical change in the way people learn. Within cyberspace, everyone has the same possibilities of exploration-a "land" where travel times are calculated only on the basis of connection speed and information is chosen in accordance with the specific interests of the users [5].

The visitor has changed the way to go to the museum. A real visit of the museum is now anticipated by his virtual archetype since the visitor explores information on the museum using the web and then decides whether to physically go to visit the museum. 
Museums and administrations have had to adapt their offer to changes in the company's request, opening their doors to new channels of communication.

The communication tools are, therefore, the basis for structuring tourism flows at the local level. Museums, archaeological sites, and valuable architectures merge into a network of systems that are important for the economy and for the growth of tourism in the territory, given that the virtual is an amplification of and a useful tool for spreading knowledge and the existence of heritage. It cannot aspire to be a substitute for the emotional experience of a real visit.

In this sense, it is important to give visibility to widespread heritage and to those monuments that remain hidden not only physically but culturally overshadowed by the fame of the giants of mass tourism - tourism that bases its foundations on the historical itineraries always furrowed by the Grand Tour. To redirect the tourist flows of the territory into a territorial management system that embraces all present patrimony and gives voice to those works that are less visible and less known.

This kind of patrimony is not less important or less "valuable" compared to others inflated by the massive tours, follows routes that have been consolidated for years.

It is important to underline how, with the advent of the digital age, the transposition of reality into virtual reality is not based on the search for representation in the most truthful way of the existing object. The three-dimensional object does not want to be the substitute replica of the existent but a means, a tool for taking advantage of and disseminating the world heritage culture. As in any type of representation from freehand drawing to vector drawings, even the three-dimensional drawing brings with it the limits of representation that means the visualized object will never be the identical copy of the real object.

For several years now, the experimental laboratory, DAda-LAB, of the University of Pavia has proposed a methodological analysis of documentation with the same purposes of protection, enrichment and recovery, but applied to different case studies ranging from the macro scale at the territorial level studying the relationship between landscape and buildings, to the more detailed scale of analysis that it is starting from the individual building arriving in the most detailed scale regarding the single digitalization and cataloging of findings and artifacts. The passage of the scale from three different levels of detail from the landscape to the macro scale of the architectural building arriving at the micro scale of the exhibit first required the application of different tools for data acquisition and needs to set up different analysis methodologies. The final objectives, even if achieved through different methods of data acquisition and processing, had a common goal: the possibility of managing the data acquired within a global network, a digital database for widespread heritage, defining a tool that puts the museum (container of art) [6] in dialogue with its content, the museum object. The activities of the laboratory are concentrating both towards the realization of a methodological process of the digitization of the museum space (container) towards innovative forms of use of the museum space, and the study of a methodological process of the acquisition of museum collections, generating new visiting experiences in the digitized space. The development of the systems of interaction between the real and the virtual with the aim of relating, in the information system, the single object or work of art with the formal, technological and constructive qualities of the architectural complex from which the element was perceived. The project aim is in line with the H2020 objectives; the laboratory is the leader of a EU Horizon 2020-R\&I-RISE-Research \& Innovation Staff Exchange: PROMETHEUS-PROtocols for information Models librariEs Tested on HEritage of Upper Kama Sites recently approved by the European Commission, dedicated to the definition of data acquisition and management methodologies for the creation of information models functional to the management and enhancement of Cultural Heritage Routes (CHR). The passage from the urban scale to that of the single object required the definition of environmental levels for a reasoned construction of archives shareable on the global network. 


\section{Study Experiences on Widespread Heritage among Remote Territories}

Among the experiences carried out, it is appropriate to select some projects that have the construction of cultural identity through procedures for the enhancement and accessibility of cultural heritage through digital databases as their objective. For the macro scale case of study, the creation of a database for the documentation of different architectural complexes that have great historical and artistic value, which are scattered far from the major tourist routes within the vast Russian territory of Upper Kama, in the Perm region, has been chosen. This territory is characterized by historical settlements, rich in industrial and religious architectures that are in a poor state of preservation today.

These complexes are the evidence of a cultural tradition between Europe and Asia, now threatened by the disappearance of such monumental systems, decorative languages and constructive traditions. These architectural complexes are located in areas difficult to access and are therefore destined, due to negligence and degradation, to the consequent loss of historical memory [7].

The project highlighted the need to plan a coherent interconnection system through the development of thematic itineraries among the various historical architectural settlements present throughout the region.

This territory, almost as big as Italy, extends from the Komi territories to the north to the provinces of Perm and Samara to the south.

The conditions under which most of the architectures, neglected in the Soviet era and aggravated by the difficult hydrogeological conditions, make these abandoned places peripheral points of tourism, frequented and often known only by local inhabitants [8]. During the summer of 2018, a first survey campaign was carried out which saw the analysis and acquisition of 14 churches (Figure 3) spread across the region.

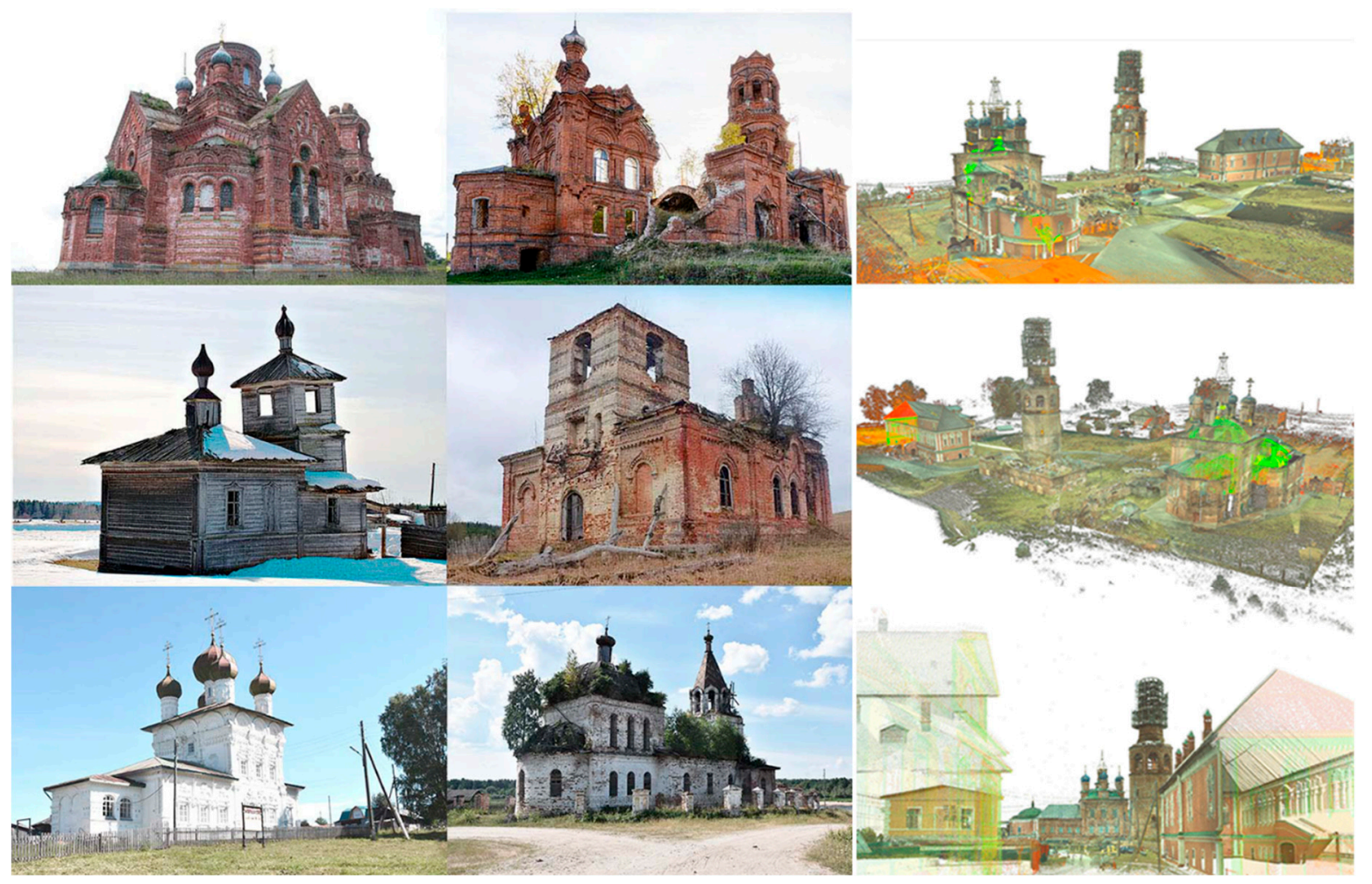

Figure 3. On the left some examples of religious architecture dispersed in the Upper Kama Region on the right some point cloud views from the laser scanner acquisition of the Usolye complex.

The analysis has been subdivided into different levels of investigation. The first one has foreseen the study of the church in relation to the landscape context. The second level of investigation has been 
focused on the documentation of the entire religious building (Figure 4). For each religious complex, through the use of laser scanner instruments (FARO S150 cam), a survey campaign has been planned of the external surfaces and of the internal rooms of the individual churches where access is possible. The laser survey was integrated through the inspection and acquisition of the roofs through the use of a drone (Phantom DJI) for aerial photogrammetric shooting and a detailed photographic campaign that included both the cataloging of the individual construction details and the brick decorations and a series for processing of the photogrammetric model (SfM) in order to study and create the three-dimensional virtual church.

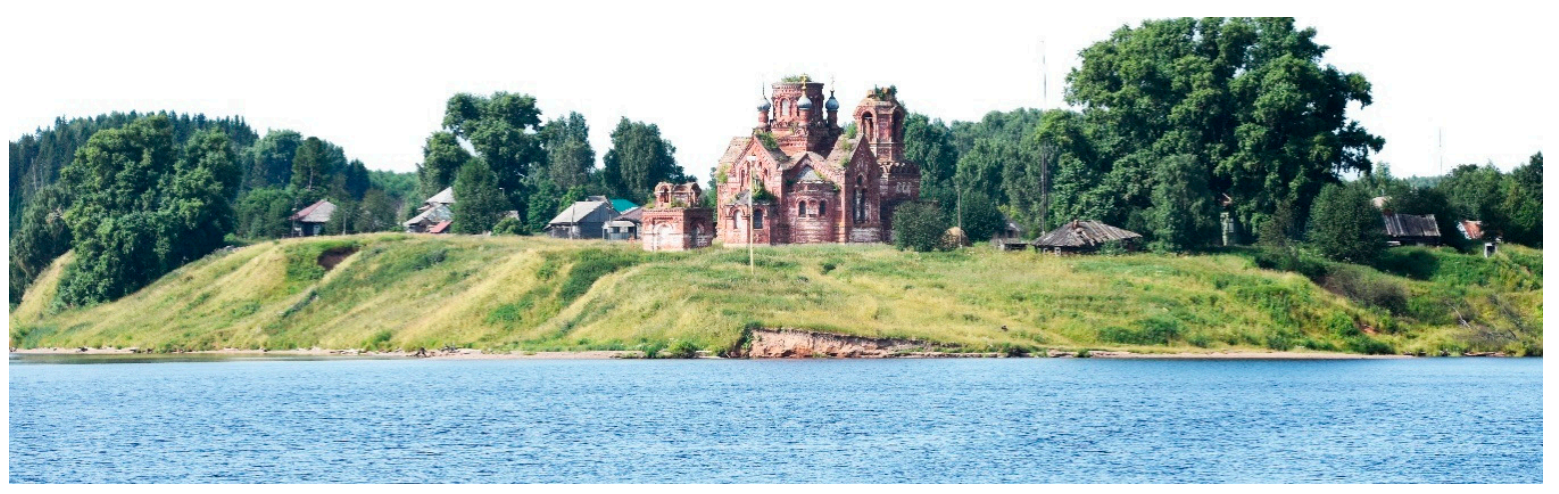

Figure 4. View of the St. Peter and Paul church in Taman. August 2018. These monumental complexes were founded between the seventeenth and nineteenth centuries, under the will of the most important merchant families of Imperial Russia. They are the synthesis between religious and residential architecture, promoted at that time by Russian merchants.

In order to recreate a virtual visiting path, a $360^{\circ}$ camera (Ricoh Theta 360) was used to create a virtual tour of the individual religious complexes. Through a path made of links between a series of spherical photos enriched with interactive contents, these models (Figure 5) promote not only the dissemination of remote and difficult to access areas, but are also inserted into a general three-dimensional model of representation at the territorial level, becoming information databases' specific points of access to information about the individual elements that characterize the territory of Upper Kama. The collection and the analysis of these data with the historical document and images even allow the planning of a hypothetical virtual restoration in order to give new life to these complexes that have been abandoned for too long. 

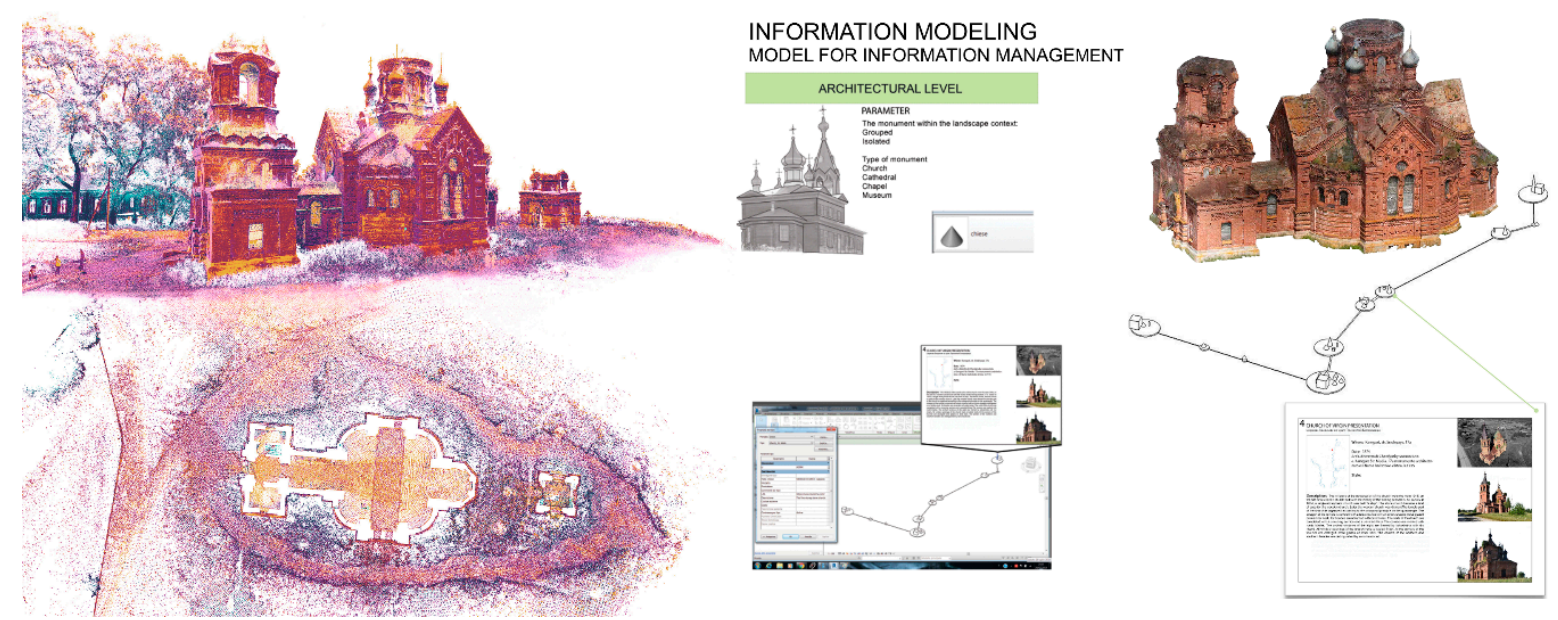

Figure 5. On the left some views from the 3D model point cloud of St. Peter and Paul church in Taman obtained with the laser scanner survey. On the top right a 3D textured model of the church made with SfM detection. On bottom right the parametric structure of a model of connections that explicates the possibilities of remote interaction between the different elements present on a widespread territory.

\section{Reconstructing the Lost Cultural Landscape through Digital Databases and Archives}

Microscale investigation analysis has studied the acquisition methodologies for the three-dimensional reproduction of the museum collection. The collection (Figure 6) used for the analysis was some of the ethnographic findings of the population of the natives of the great North American plains, which are divided between the various private and non-museum collections.

The virtualization of the historical heritage does not matter if it is a single find or architectural systems; it implies the transition to a hypertext, a reticular structure of information that represents the result of an analysis and a synthesis of the forms [9]. The documentation project concerning the Native Americans findings was aimed to obtain an accurate model through a detailed survey in order to have a good copy of the finding, complete with all the information regarding the form, the materials, and the conservation status of the single object. The passage of a scale from a survey finalized to the macro building element to a representation of the decorative detail, such as the single bead or the feather that adorns the headgear, requires reflection on what type of instruments can be adapted to the accurate restoration of detail in the order of $1 \mathrm{~mm}$. 

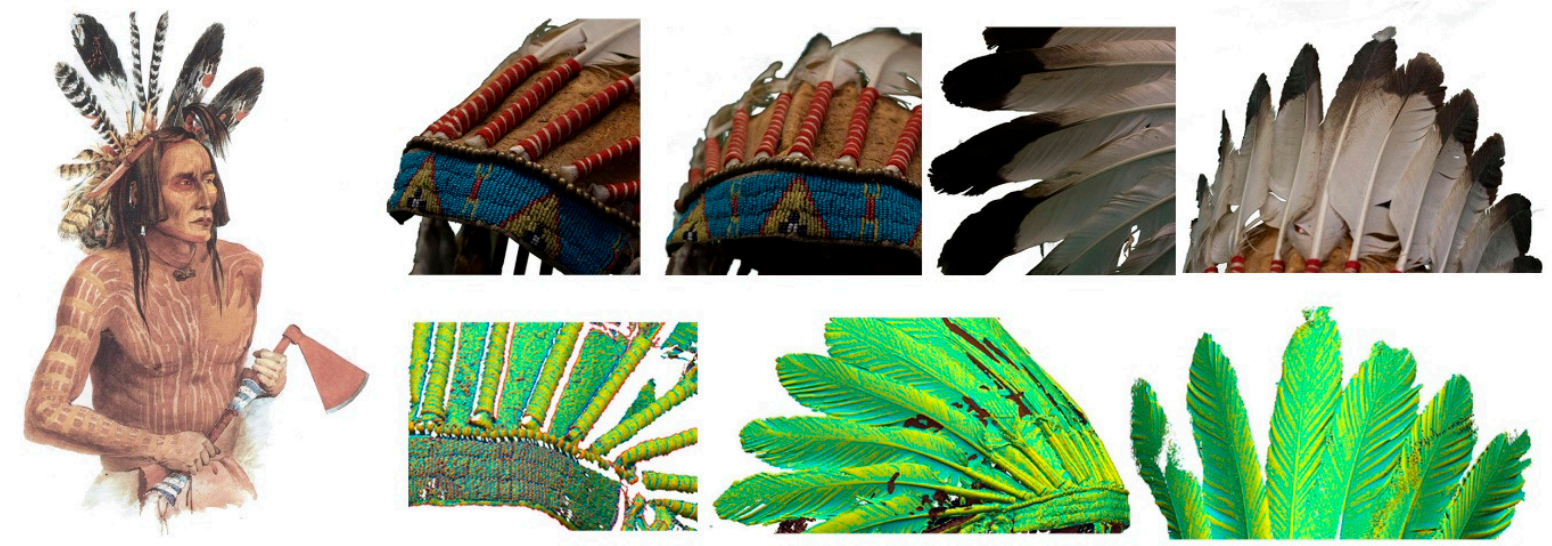

Figure 6. On the left an illustration depicting a Native American while wearing a war bonnet. On the top right some images of the bonnet used for the reasearch is a museum replication of the period around 1870. It is composed of an elk/bison calf leather of Golden Eagle (tail) red exchange fabric (trade cloth) leather, ermine, and a leather band decorated with Venetian glass beads (Murano island beads) sewn with deer tendon. Some of the detail in the 3D model of the bonnet acquired. On the bottom right the detail of the 3D model of the bonnet after the acquisition whit the structured light scanners.

In this regard, three different types of structured light scanners were compared, Eva Artec, Spider Artec and DAVID 3D, on two types of objects with different characteristics, a war bonnet and an instrument. The comparison showed that the DAVID 3D scanner led to more accurate restitution of detail than the other two models which caused various inaccuracies due to the phenomenon of light refraction.

The three-dimensional data of the individual objects were elaborated (Figure 7) through mesh surface management programs (software Geomagic Design X). They were optimized through a correction of the defects and gaps present on the surface, and a decimation of the high number of polygons of the surface, paying attention to not lose all the information related to the detail necessary to describe the formal qualities. In this way, the final model results are manageable within the specific three-dimensional visualization software. 


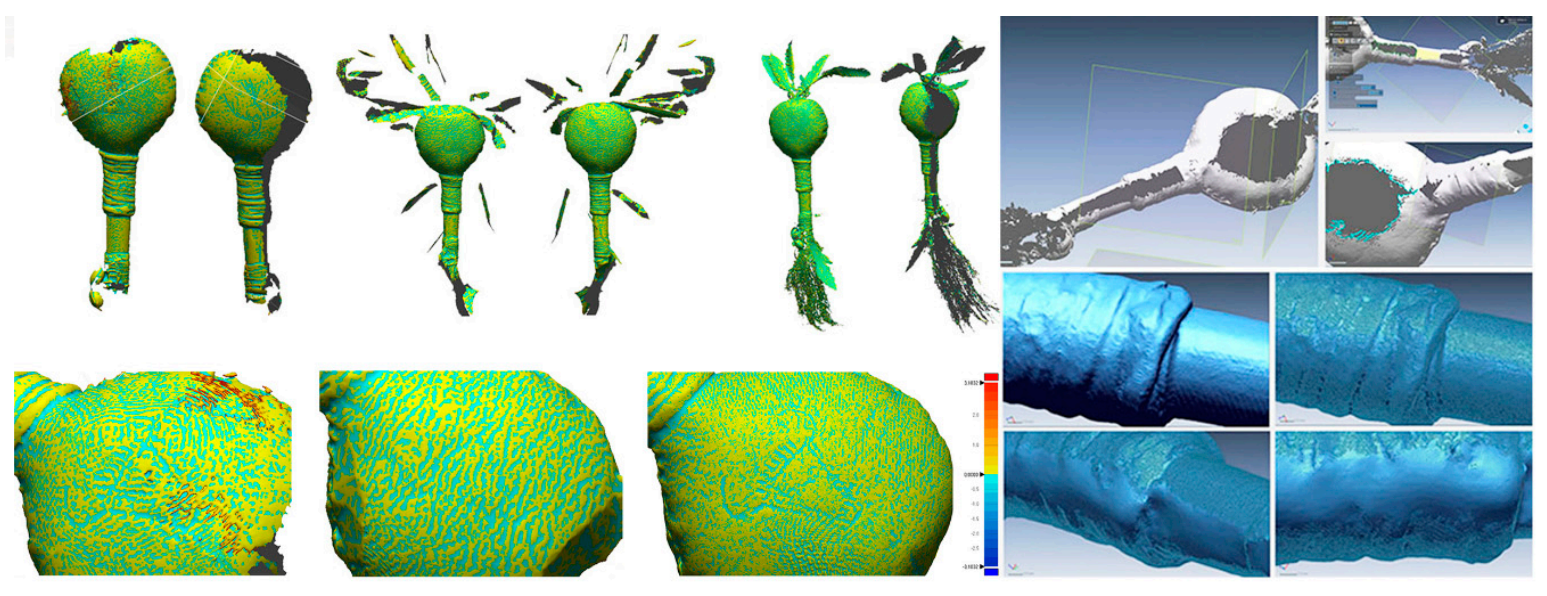

Figure 7. Comparison between models is shown from left to right: Artec Spider, Artec Eva, David-3D. Below is the detail of the acquisition of the incisions: Artec Spider, David-3D, Artec Eva. The parts of the incisions were highlighted in blue by the software used for the analysis of the Geomagic Design $X$ meshes, which determined a depth of about $0.5 \mathrm{~mm}$. The parts highlighted in blue, instead of the one highlighted in red, in the model generated by the Spider indicate an alignment error of the scans $(3 \mathrm{~mm})$. On the right, the operations of the optimization of the mesh and the result of the reconstruction of the handle of the maracas, the image shows how in the points in which it was reconstructed, the polygonal mesh is more simplified.

In addition to the simulation of the geometric forms, it was possible to associate all the descriptive historical information to the copy of the object, which, inserted into digital databases, preserves its historical memory. The creation of these interactive databases and the development of virtual museum systems also make possible the promotion of peripheral tourism centers, which are little known or difficult to access [10].

The models obtained in this way from the acquisition to the data optimization phase are reliable from a metric point of view and complete with geometrical and colorimetric information. They can be used to define an information database on the morphological qualities of each work. Specifically, using reverse modelling software, it is possible to set cutting planes and to separate the individual objects to obtain dimensional drawings relative to the external volume and the dimension of the object.

In this form, the same models can be prototypes, printed in 3D by reconstructing 3D objects as much as faithfully as possible to the original in order to compose exhibitions and functional arrangements in the story of the culture of the natives.

With this in mind, there is the possibility of indexing the database to connect it to an international databases, connected for example to the museum system. The aim is to be able to create an index the models that would become the guiding elements of the entire information system. Parametrization of the models proved to be the most appropriate way to connect different types of information (including multimedia files) to the virtual object. At this point, two paths have been outlined: the first concerned importation of the models obtained by the acquisition phase within the Autodesk Revit software, to associate to each portion of the model (decomposed according to a semantic reading of its construction components) different information deriving from the database.

In this way, however, a truly parametric model has not been obtained. This is to the advantage of a model that fully describes the physical characteristics of the object represented, which is both a limitation due to the use of a discrete system deriving from the point cloud and the mesh modelling constraints - both in terms of file size and, therefore, management of a database composed of a very large number of elements and in terms of functionality of the information system.

Therefore, we tried to parameterize the model to construct a virtual stereotype that could be associated with the mesh model to uniquely identify the work with all its formal three-dimensional 
structural characteristics and thus, through a symbolic representation of the object, to define groups and families of elements belonging to a specific collection containing at the same time all the information of the database including the reality-based model generated by the survey (Figure 8).

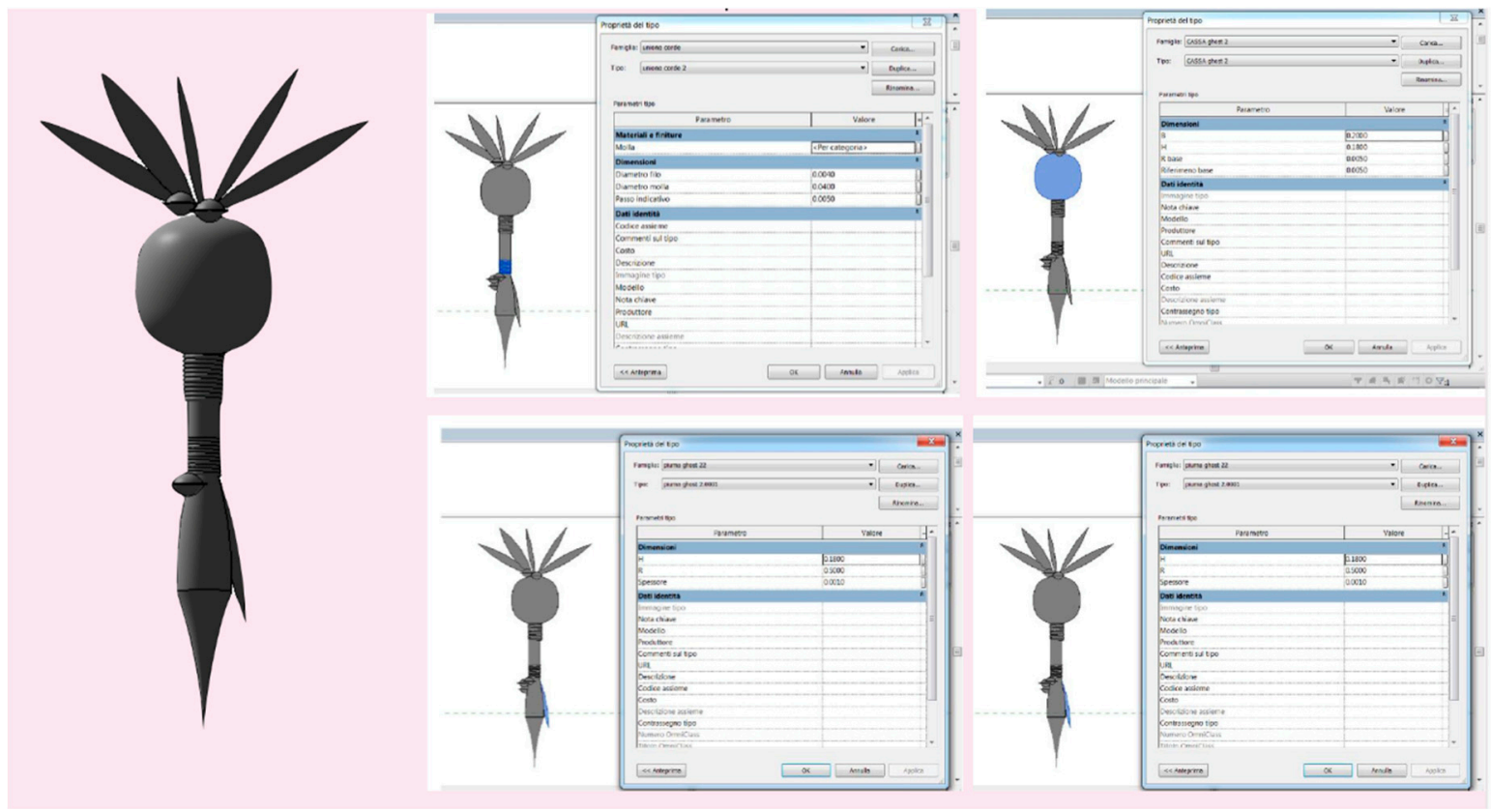

Figure 8. On the left the 3D model of the rattle. On the right comes views describing that all elements of the virtual stereotype of the rattle could be questionable (highlighted in blue).

The individual components of the artifact have been modelled by creating ad hoc families for each element in order to constitute a parametric library through which they can quickly index the elements that make up the work. This makes possible further study, even at a distance, of works otherwise difficult to investigate.

Different kinds of information and media, such as video, audio tracks, photographs, descriptions and data sheets, were associated with a single object of the collection. These files constitute an archive made up of the large number of traditional materials of the Native American populations that are dispersed among numerous private collections today [11]. The data were collected on a website that, in addition to the history of the Native Americans, allows a visit to the digitalized collection (Figure 9). 


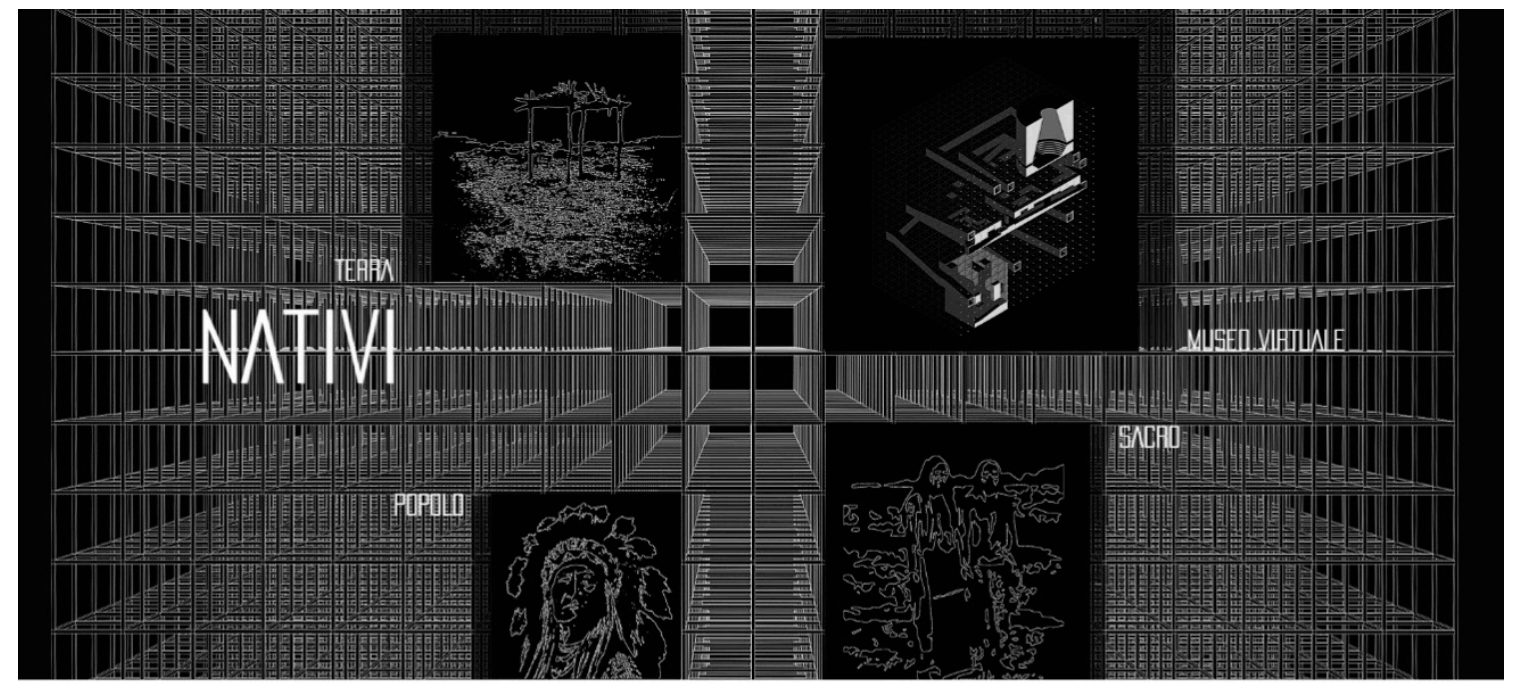

Figure 9. Cont.
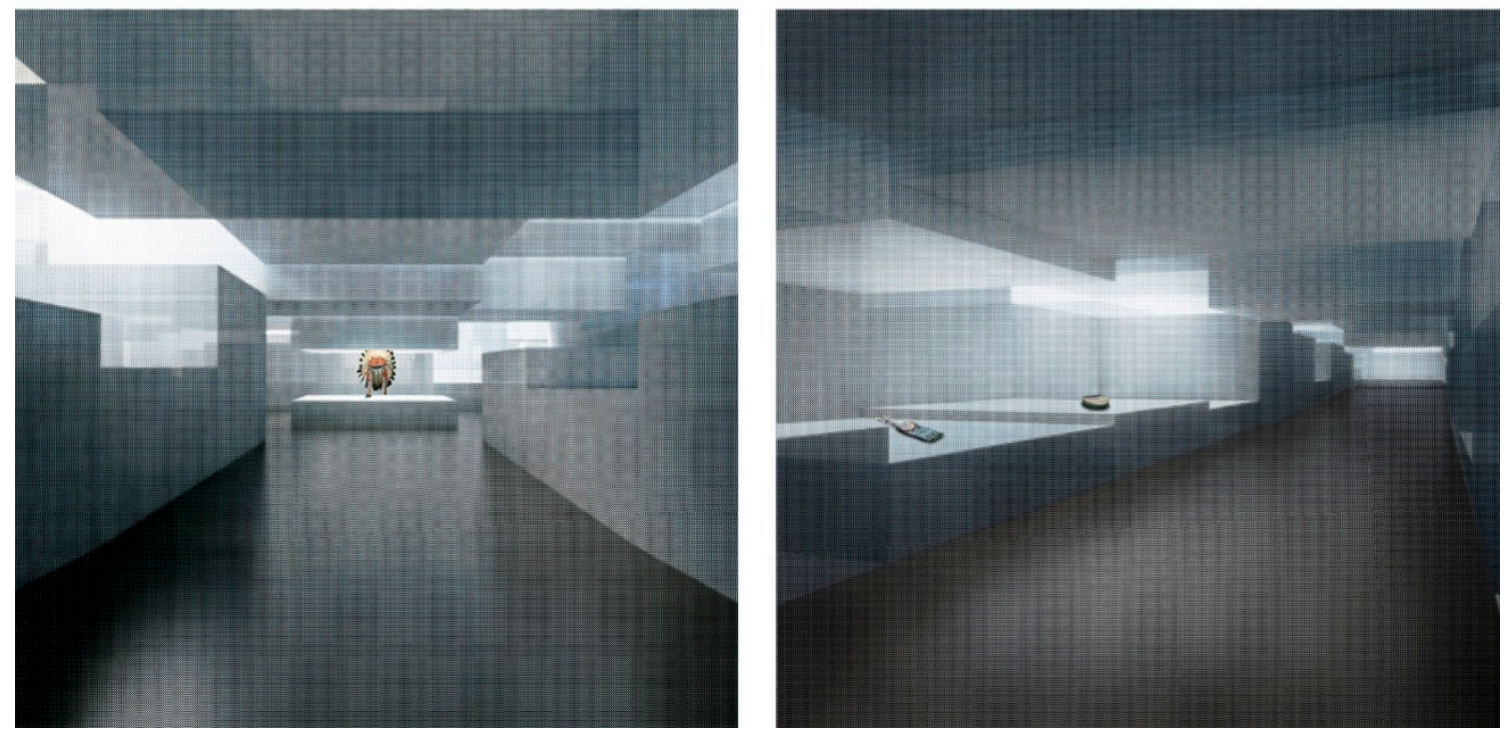

Figure 9. Views of the development of a website of the virtual museum (on the top) and (on the bottom) two views of the exhibition hall 3D model of the virtual exposition of the Native American collection (Developed by Stella Giannini).

\section{Conclusions}

A virtual copy of the findings inserted into a complex database full of information and accessible through virtual platforms, such as local-based, geo-referenced, and support systems for museum complexes, encourages combining content and containers within a virtual space; it is now ever more implementable. The dialogue between the general models of architectures and the data relating to the individual elements that compose them amplifies the potential of these databases due to the development of remote fruition systems, which simplify the search operations. Artworks, objects and architectures have a second opportunity for development by bringing back the original artifacts in their architectural context virtually, giving rise to movements, sonority, and explicit content that enrich the virtual experience and facilitate interpretation in the real system. Through virtual 3D models, the artwork regains dignity and a function aimed at the documentation, conservation and historical 
dissemination of cultural heritage inside museum systems, giving way to the virtual the possibility of replicating reality in a simplified form in order to create tools for documentation and teaching, through the development of serious games, apps and the digital printing of prototypes.

Through the use of different methodologies, an analogous result was determined, i.e., the virtual reproduction of the object and the insertion of this within a complex database, rich in information accessible through virtual platforms: local-based fruition and geo-referencing systems in the Russian case and support systems for museum complexes. This does not exclude the possibility of establishing a dialogue between the two results through the implementation of the general models of architectures with the data related to the single valuable elements that compose them.

The methodology for the acquisition of Native Americans collections can be applied to all those works that, by abandoning their place of origin, have been closed inside museums. This makes it possible to virtually bring back the original artifacts in their native architectural context, thus enriching the perception of the work, which regains its original function, and of the architecture, which is covered with its decorative system and restores its original image.

Author Contributions: The editorial responsibility of the paragraphs is recognized to: S.P. for the paragraphs 1 and 4, and A.D. for the paragraph 2 and 3. Conceptualization, S.P. and A.D.; methodology, S.P. and A.D.; software, A.D.; validation, S.P. and A.D.; formal analysis S.P. and A.D.; investigation S.P. and A.D.; resources, S.P. and A.D.; data curation, S.P. and A.D.; writing-original draft preparation, S.P. and A.D.; writing-review and editing, S.P. and A.D.; visualization, S.P. and A.D.; supervision, S.P. and A.D.; project administration, S.P.

Funding: The realization of international summer schools is founding by University of Pavia and Perm National Research Polytechnic University. The project "PROMETHEUS-PROtocols for information Models librariEs Tested on HEritage of Upper Kama Sites", is financed by the EU Horizon 2020 programme-RISE-(g.a. n $\left.{ }^{\circ} 821870\right)$ with the collaboration between Universities, (University of Pavia, Italy; Universitat Politècnica de València, Spain; Perm National Research Polytechnic University, Russia) and enterprises (EBIME, Spain; SISMA, Italy). The research project about the documentation of Native American art crafts was made possible thanks to the found of the experimental laboratory DAda Lab of the University of Pavia and the Wambli Gleska Association.

Acknowledgments: The research project concerning the documentation of the architectures of the Upper Kama Region was made possible thanks to the collaboration between the Dada Lab of the University of Pavia and the Perm National Resarch Polytechnic University and thanks to the contribution of the Russian Academy of Painting, Sculpture and Architecture, the Stroganov Chambers Historic Architectural Museum and the administration of Usolye. The research project about the documentation of Native American art crafts was made possible thanks to the collaboration between the experimental laboratory DAda Lab of the University of Pavia and the private collector Sergio Susani.

Conflicts of Interest: The authors declare no conflict of interest.

\section{References}

1. Mario, G.; Aurélien, P. Mondi Virtuali:Benvenuti nel Futuro Dell'umanità; Castelvecchi editore: Roma, Italy, 2006; 350p, ISBN 8876151095.

2. Palestini, C.; Basso, A. Oxymorons of the virtual museum. Experimentaton through the representation. DISEGNARECON 2016, 9, 6.1-6.15.

3. Verdiani, G. Ricostruire nel Virtuale e nel Reale, Esperienze tra Musei e Divulgazione; Jasink, A.M., Dionisio, G., Eds.; Firenze University Press: Firenze, Italy, 2016; pp. 195-208.

4. Comunicato Del Ministero Degli Affari Esteri Del 25 Febbraio 2009. L'italia per L'IRAQ-la Riapertura del Museo di Baghdad. Available online: http:/ / www.beniculturali.it/mibac/export/MiBAC/sito-MiBAC / Contenuti/Ministero/UfficioStampa/ComunicatiStampa/visualizza_asset.html_1509809224.html (accessed on 12 November 2018).

5. Georgopoulos, A. Data Acquisition for geometric Documentation of Cultural Heritage. In Mixed Reality and Gamification for Cultural Heritage; Ioannides, M., Magnenat-Thalmann, N., Eds.; Springer: Cham, Switzerland, 2017; pp. 29-73.

6. Genovesi, E. Simulazioni per un progetto: Il museo diffuso e il sistema museale. In Musei e Pachi Archeologici, IX Ciclo di Lezioni Sulla Ricerca Applicata in Archeologia (Certosa di Pontignano 1997); Francovich, R., Zifferero, A., Eds.; All'insegna del giglio: Firenze, Italy, 1999; pp. 105-143. 
7. Brumfield, W.C. The Architectural Heritage of Solikamsk and the North- ern Districts of Perm Province. Cah. Slaves 2008, 10, 317-355. [CrossRef]

8. Parrinello, S.; Cioli, F. Un progetto di recupero per il complesso monumentale di Usolye nella regione della Kama Superiore. Restauro Archeologico 2018, 1, 92-111.

9. Lévy, P. Il Virtuale; Raffaello Cortina Editore: Milano, Italy, 2014.

10. Guidi, G.; Russo, M.; Beraldin, J.A. Acquisizione 3D e Modellazione Poligonale; McGraw-Hill: Milano, Italy, 2010.

11. Parrinello, S.; Dell'Amico, A. The traditional crafts of the native Americans Procedures of survey and documentation for the creation of informative systems 3D. In Proceedings of the Conference 3D Modeling and BIM Nuove frontiere, Roma, Italy, 18-19 April 2018.

2019 by the authors. Licensee MDPI, Basel, Switzerland. This article is an open access article distributed under the terms and conditions of the Creative Commons Attribution (CC BY) license (http://creativecommons.org/licenses/by/4.0/). 